\title{
ENCONTROS COM A ATIVIDADE: A EXTENSÃO DO DIÁLOGO SOBRE TRABALHO, GESTÃO E SAÚDE MENTAL
}

\author{
Wladimir Ferreira de Souza ${ }^{1}$ \\ Alessandra Lopes Alves Smaczylo Rodrigues ${ }^{2}$ \\ Thaciane Assis dos Santos ${ }^{2}$
}

RESUMO: Este trabalho apresenta os resultados parciais do projeto de extensão universitária que vem sendo desenvolvido, a partir de 2014, por uma equipe de professores e alunos do curso de graduação em Psicologia de uma universidade pública do estado do Rio de Janeiro. O projeto aborda temáticas decorrentes das relações entre trabalho, gestão e saúde mental e está relacionado com as atividades de ensino e pesquisa dos professores participantes. Realiza-se em parceria com um Centro de Referência em Saúde do Trabalhador (CEREST), unidade de saúde que abrange quatro municípios do estado do Rio de Janeiro. A discussão baseia-se na produção teórica e metodológica presente no campo da saúde mental relacionada ao trabalho.

PALAVRAS-CHAVE: Psicologia. Trabalho. Gestão. Saúde mental.

Meetings with the activity: extending the discussion about work, management and mental health

\begin{abstract}
This study aims to present the partial results of a university extension project that has been developed, since 2014, by a team of professors and undergraduate students of a public university in the state of Rio de Janeiro, Brazil. The project describes the relation between work, management and mental health and is related to the teaching and research activities of professors that participated in this project. It has been developed in partnership with the Reference Center for Worker's Health, a health unit that gives assistance to four cities in the state of Rio de Janeiro, Brazil. The discussions are based on the theoretical and methodological production in the field of mental health related to work.
\end{abstract}

KEYWORDS: Psychology. Work. Management. Mental health.

\section{INTRODUÇÃO}

Desde março de 2014, um projeto de extensão universitária que aborda as relações entre trabalho, gestão e saúde mental vem sendo desenvolvido no Instituto de Psicologia de uma universidade pública do estado do Rio de Janeiro. Esse projeto foi proposto pelo coordenador, professor adjunto da universidade, e foi aprovado pelo Departamento de Extensão da Sub-reitoria de Graduação da referida instituição.

\footnotetext{
${ }^{1}$ Doutor em Psicologia Social pela Universidade do Estado do Rio de Janeiro, onde atua como professor adjunto do Instituto de Psicologia (souzalandi@uol.com.br).

${ }^{2}$ Graduandas em Psicologia na Universidade do Estado do Rio de Janeiro (alesmaczylo@hotmail.com; thaciane.assis@hotmail.com).
} 
A proposição do projeto partiu de algumas constatações. Uma delas é que ainda não se tem, no Brasil, um conhecimento suficiente e conclusivo para uma compreensão mais aprofundada e para intervenções mais eficazes sobre a problemática citada, embora os chamados transtornos mentais e comportamentais relacionados ao trabalho estejam entre as principais causas de afastamento do trabalho, segundo dados do Anuário Estatístico da Previdência Social (BRASIL, 2012a). Essa constatação é corroborada por diversos textos científicos, como, por exemplo, aqueles publicados no dossiê específico sobre o tema na Revista Brasileira de Saúde Ocupacional (RBSO, 2010; 2011).

Outra constatação pode ser encontrada também em documentos emitidos pelo Conselho Federal de Psicologia (CFP/CREPOP, 2008; CFP, 2010), que assinalam que a formação dos psicólogos (salvo raras exceções) não contempla, de modo suficiente, uma abordagem crítica que considere o vínculo entre os processos de subjetivação, o trabalho, a gestão e as formas de gerenciamento e a ocorrência de casos de sofrimento psíquico/adoecimento mental, o que leva muitos profissionais a negligenciarem a relação desses fatores em suas práticas. Em muitos casos, individualiza-se o problema, pondo-se ênfase na psicopatologia, deixando-se de lado o fato de que o trabalho é uma atividade coletiva fundamental para os humanos e que jamais é neutro em relação à saúde, podendo, sim, ser patogênico, mas não somente isso, pois a relação dos sujeitos com o trabalho pode ser fator de prazer, satisfação, criatividade e realização de si (DEJOURS, 2004; 2012).

Verifica-se, assim, que, no campo social mais amplo, a minimização e até mesmo a negligência em torno da importância da relação entre o processo de trabalho e os processos de saúde e adoecimento/sofrimento psíquico apontam para a necessidade e a premência de formação mais específica para os alunos de Psicologia e de explanação e discussão dessa temática com diversos atores sociais, para além do âmbito acadêmico.

Desse modo, buscando transpor essa discussão para além dos muros da universidade, propôs-se uma parceria com uma instituição que lida com a saúde dos trabalhadores (dedicada e sensível ao debate sobre a temática), por meio da qual fosse possível envolver uma diversidade de atores. A instituição parceira escolhida foi o Centro de Referência em Saúde do Trabalhador (CEREST) de um município do estado do Rio de Janeiro.

Os CEREST são unidades de saúde pública de abrangência estadual, regional ou municipal pertencentes ao Sistema Único de Saúde (SUS). Oferecem suporte técnico e científico e desenvolvem um conjunto de atividades, que incluem vigilância, promoção, proteção e prevenção no campo da saúde do trabalhador. As ações, nesse campo, são de responsabilidade do SUS, conforme preconiza a Constituição Federal e a Legislação Infraconstitucional (BRASIL, 2009; 2012b). O CEREST parceiro do projeto tem abrangência regional, sendo responsável pelas ações no município sede e em outros três de sua área de abrangência.

Em reunião com a direção do CEREST e com os membros dos diversos segmentos dos municípios que o integram, houve interesse pela parceria com a universidade. Um documento foi emitido pela direção, declarando o compromisso e o interesse para que o projeto se desenvolvesse lá.

Atualmente, integram a equipe, alunos da graduação em Psicologia (que passaram por uma entrevista de seleção) e três professores do Instituto de Psicologia da universidade, dentre eles o coordenador do projeto. Pretende-se fazer uma seleção, em breve, para admissão de novos alunos no próximo semestre letivo. 
Por intermédio do CEREST, profissionais de saúde e de educação, que atuam na área de saúde do trabalhador, além de outros profissionais com função de "gestão" (gerenciamento) no SUS (diretores, coordenadores, chefes de equipes etc.) e membros do segmento de usuários (participantes dos conselhos municipais de saúde ou dos conselhos gestores das unidades de saúde) engajam-se nas atividades oferecidas, representando diversos órgãos da sociedade civil, como associações de moradores e sindicatos de trabalhadores dos seus respectivos municípios.

\section{METODOLOGIA}

Antes da implementação, realizaram-se reuniões com os membros dos diversos segmentos participantes do CEREST para apresentação do projeto e preparação para o início das atividades. Nessas reuniões, procedeu-se a análise conjunta da demanda e da pertinência do projeto para aquela realidade específica, além da validação consensual da necessidade de sua execução. As reuniões preparatórias ocorreram nas datas abaixo:

1. 13 de março de 2014: apresentação do projeto pelo coordenador aos profissionais do CEREST e do Programa de Saúde do Trabalhador (PST) na reunião de elaboração do plano de ação de 2014 da saúde do trabalhador do município sede. Nessa reunião, as atividades do projeto foram incluídas no plano de ação;

2. 20 de março de 2014: apresentação do projeto pelo coordenador aos representantes de diversos segmentos dos quatro municípios abrangidos pelo CEREST. Nessa reunião, incluiu-se a apresentação do projeto na programação do Encontro Regional de Saúde do Trabalhador.

Nessas ocasiões, foi informado aos participantes que o projeto propõe a realização de múltiplas ações, como oficinas, palestras, apresentações, exposição de pôster, cursos e encontros. Informou-se, também, que cada atividade destina-se a públicos específicos, considerando a inserção em cada segmento, a área, o nível de formação e o âmbito de atuação. De acordo com esses fatores, são escolhidos os temas e as formas de transmissão do conteúdo e definidas as técnicas a serem empregadas (juntas ou separadamente): aulas expositivas, dinâmicas de grupo, leitura e discussão de textos e a construção de material coletivo acerca da temática debatida.

As atividades previstas contemplam as seguintes ações extensionistas:

a) Participação da equipe (em palestras, oficinas e/ou exposição de pôster) nos Encontros de Saúde do Trabalhador (regional e estadual) preparatórios para a Conferência Nacional de Saúde do Trabalhador;

b) Oficina "Dialogando sobre as relações entre saúde mental e trabalho", destinada aos profissionais de saúde e educação em geral e aos usuários do SUS dos municípios abrangidos pelo CEREST;

c) Curso de "Iniciação às abordagens em Saúde Mental relacionada ao Trabalho", destinado aos profissionais de saúde em geral; 
d) Curso de Atualização "Transtornos mentais e comportamentais relacionados ao trabalho: pistas para reconhecer e intervir", destinados aos profissionais de saúde mental e de saúde do trabalhador;

e) Encontro ou Seminário: "Trabalho, gestão e saúde mental: um encontro útil e necessário", destinado aos profissionais de saúde e educação em geral e aos usuários do SUS dos municípios abrangidos pelo CEREST.

As ações são divulgadas nos diversos contatos presenciais (em reuniões e eventos), por meio de cartazes e por meio eletrônico. No dia da atividade, os inscritos assinam a folha de presença. Para obtenção do certificado de participação nas atividades, deve-se ter, no mínimo, $75 \%$ (setenta e cinco por cento) de frequência.

No que tange à formação dos alunos de graduação envolvidos no projeto, ocorrem reuniões, nas quais se faz a análise crítica e discussão de textos propostos anteriormente para leitura, preparam-se as atividades voltadas para o público-alvo do projeto e orientam-se os alunos para participação nas atividades propostas. Além disso, nas disciplinas da graduação ministradas pelos professores que integram o projeto, a temática é trabalhada e debatida por meio de aulas expositivas dialogadas, leitura e discussão de textos e realização de trabalhos individuais ou em grupos.

\section{MATERIAL}

Os alunos, os professores e os inscritos nas atividades têm acesso ao referencial teóricometodológico que embasa as ações, artigos e capítulos de livros, disponibilizados para leitura e discussão, tanto no formato impresso como por meio da Internet.

Nas exposições dos membros da equipe aos participantes inscritos nas atividades propostas, são utilizados diversos recursos, dentre eles auditório ou sala de aula, pôster científico, computador portátil (notebook), projetor multimídia, tela de projeção, quadro branco, textos científicos e materiais de papelaria.

\section{DISCUSSÃO}

O campo da saúde mental relacionada ao trabalho (SMRT) (SELIGMANN-SILVA, 2011; PAPARELLI; SATO; OLIVEIRA, 2011) engloba a discussão acerca das relações entre os processos de trabalho e os processos de saúde-adoecimento dos indivíduos, em que se encadeiam múltiplos fenômenos que podem propiciar maior vitalidade ou maior fragilização da saúde. Entre esses fenômenos estão compreendidas as forças políticas e sociais que podem favorecer ou fragilizar a saúde dos seres humanos nas relações de trabalho.

Seligmann-Silva et al. (2010a, p. 185) destacam que:

embora a relação trabalho e saúde tenha sido relatada desde a Antiguidade, as primeiras abordagens formais desta relação tiveram início na Europa, no 
século XIX, com a criação da Medicina do Trabalho e a implantação dos serviços médicos dentro das empresas.

As autoras assinalam que, sobretudo nas décadas de 1990 e 2000, as repercussões psíquicas do trabalho passaram a ganhar espaço nos consultórios e a serem reconhecidas no Brasil como estando relacionadas a ele, conforme portaria e decreto emitidos pelo Ministério da Saúde e pelo Ministério da Previdência Social.

Não obstante esses fatos apontados, as autoras acrescentam que o pensamento tradicional das áreas de Medicina do Trabalho, de Saúde Ocupacional e de Psicologia dá pouca atenção para o trabalho como mediador de integração social e para a sua importância na constituição da subjetividade das pessoas, bem como para o fato de que esses aspectos são influenciados por mudanças (ou rápidas transformações) no mundo laboral causadas pela globalização financeira e precarização social mundializada (SELIGMANN-SILVA et al., 2010b). Outro aspecto importante que vem sendo negligenciado refere-se às repercussões das inovações tecnológicas e das novas formas de gestão e controle do trabalho.

Para Seligmann-Silva (2011), Medicina do Trabalho, Saúde Ocupacional e Saúde do Trabalhador $^{3}$ são mais que três disciplinas voltadas para um mesmo objeto: constituem três abordagens distintas.

Historicamente, o consenso tradicional aponta que surgiu primeiro a Medicina do Trabalho e que a mesma abrigou a análise das causas e danos referentes aos acidentes de trabalho, a investigação de agentes etiológicos de patologias laborais e a implementação de medidas de proteção aos trabalhadores. [...] A denominação Saúde Ocupacional é utilizada predominantemente nas empresas e geralmente é adotada segundo uma lógica positivista voltada à linearidade do tipo causa-efeito, sem considerar a complexidade que a dimensão social e a dimensão subjetiva introduzem nas questões da saúde. [...] A designação do campo da saúde que diz respeito à saúde em sua relação com o trabalho varia internacionalmente $\mathrm{e}$ de acordo com distintas visões de ordem política e ideológica. No Brasil, o Sistema Único de Saúde (SUS) incorporou a designação Saúde do Trabalhador a partir da ideia de que essa denominação corresponde ao objetivo essencial dessa área (SELIGMANN-SILVA, 2011, p. 110-111, grifos da autora).

Mesmo com o objetivo essencial traçado e com o suporte na legislação, a área de saúde do trabalhador ainda se ressente de maior embasamento e capilaridade, conforme demonstra Seligmann-Silva (2011). Para a autora, por exemplo, nessa área, a Psicodinâmica do Trabalho (PDT) - uma importante abordagem do campo da SMRT (segundo ela, um campo multidisciplinar para o qual diversas disciplinas confluem) - embora algumas vezes tenha seus conteúdos incluídos no treinamento dos profissionais da área, o mesmo ainda não acontece com relação às equipes de saúde mental do SUS.

Nas empresas, o que a autora constata é que, embora muitos profissionais das áreas de recursos humanos tenham conhecimento da PDT e de outras abordagens pertinentes que possam fundamentar ações de prevenção e intervenções para promoção e proteção da saúde mental, em geral, eles não têm poder de implantar transformações amplas das situações reais de trabalho.

\footnotetext{
${ }^{3}$ Acerca das condições de emergência da Medicina do Trabalho, da Saúde Ocupacional e da Saúde do Trabalhador, sugerimos a leitura de Mendes e Dias (1991).
} 
Paparelli et al. (2011) afirmam que persistem as ideias que negam o nexo causal entre o trabalho e a saúde mental e criticam a lógica simplificadora e culpabilizante aí contida. Os autores apontam fatos que parecem indicar que "a divulgação, o debate e a discussão sobre o assunto não têm sido suficientes para mudar o quadro dos problemas de saúde mental relacionados ao trabalho" (PAPARELLI et al, 2011, p. 119).

Diante da situação relatada acima, os autores apregoam a necessidade de informar e afirmar que a saúde mental pode ser afetada pelo trabalho e relatam uma dificuldade que vêm encontrando em suas atuações como docentes: a contrariedade de alguns estudantes quando os professores apontam o processo saúde-doença como socialmente influenciado. Parecelhes que essa reação dos estudantes é motivada por duas posições em relação à compreensão da relação saúde-doença e trabalho:

\begin{abstract}
A primeira é a de que a causa última para explicar o adoecimento de trabalhadores e trabalhadoras deveria ser buscada sempre no indivíduo: em suas peculiaridades em termos físicos, fisiológicos, psicossociais e em sua história de vida no núcleo familiar anterior à vida de trabalho. A segunda posição é a adoção da naturalização da ideia de que o trabalho produz sofrimento e não pode ser mudado, como se isso fosse um destino, uma determinação inquestionável e intransponível, cujo curso seria impossível de ser modificado. (PAPARELLI et al., p. 119).
\end{abstract}

Parece-nos evidente que essas posições não estão desvinculadas de seu contexto histórico e social. Historicamente, tem-se destacado os aspectos negativos do trabalho, com base nas vivências de trabalhadores e trabalhadoras sob o modo exploratório e predatório de produção capitalista. Acrescente-se aí a inserção do Brasil no mercado capitalista, em que, segundo Athayde (2011, p. 350), "a força ideológica escravista persevera, combinando-se perversamente o clientelismo, o mandonismo, o machismo e o populismo".

Athayde (2011) acrescenta que, nessa formação social, pululam as possibilidades de adoecimento e acidentes de trabalho, sendo absolutamente necessário denunciar a nocividade, mas não é o suficiente, posto que a crítica pura e simples pode estar embasada em um referencial simplificador da questão, tornando-se um fardo que age na prática como obstáculo epistemológico para a busca de comprender-transformar.

Nesse sentido, o autor propõe uma ampla e necessária discussão, focando seu texto na interrogação sobre o "mental" presente ao trabalhar, buscando fazer considerações sobre a incorporação do trabalhador no processo de pesquisa-intervenção, por entender que ainda persistem resistências a essa participação efetiva, sendo esse um ponto de fragilidade nesse campo. Propõe, também, uma alternativa para explorar as questões acerca da saúde mental e do trabalho, com base nas relações entre os campos instituídos e denominados saúde do trabalhador e saúde mental e atenção psicossocial/estudos da subjetividade - operando mais fecundamente neste "entre", engendrando as questões pertinentes ao trabalho nesse espaço, haja vista, por exemplo, que no campo saúde mental e atenção psicossocial, a questão trabalho sequer emergiu com vigor.

Acerca do nexo causal, Athayde (2011), explorando diversos autores, lembra que a busca para estabelecer o nexo causal tem história e que alguns equívocos podem ser cometidos quando a estratégia de produção científica subordina-se ideologicamente à luta políticosindical. Tanto um modelo simplista de mera atribuição de causa-efeito quanto uma defesa da múltipla causalidade podem representar modelos em que a ênfase recai na patologia, encobrindo a dinâmica presente na relação subjetiva com o trabalho, como vêm destacando 
algumas disciplinas e abordagens extremamente fecundas, como a psicodinâmica do trabalho, a filosofia vitalista de Canguilhem e a dialética marxista (em uma visão não reducionista de Marx, que considera que os elementos do processo de trabalho são fruto da atividade de trabalho humana).

Souza (2013) aponta algumas dificuldades no campo "psi" no que concerne ao estabelecimento do nexo causal entre os transtornos mentais e os aspectos organizacionais do trabalho. Diversos fatores contribuem para essas dificuldades, sendo um deles aquele que diz respeito à identificação das situações laborais que favorecem o aparecimento e o agravamento dessas doenças.

Outros fatores são listados pelo autor e dizem respeito: a) a inexistência de uma lesão física evidenciável, na maioria dos casos; b) a desqualificação das manifestações de sofrimento, que não coincidem com as referências diagnósticas dos manuais, já que, em geral, o quadro apresenta-se como uma síndrome inespecífica, um sofrimento e uma queixa difusos, que inclui mal-estar, medo, ansiedade, depressão, nervosismo, tensão, fadiga, perda de apetite, distúrbios do sono e psicossomáticos; c) o não reconhecimento social de que alguns casos de sofrimento psíquico podem comprometer a capacidade de trabalhar; d) o fato de que o processo de adoecimento é específico para cada pessoa, o que leva, muitas das vezes, à recomendação de tratamentos individuais e pontuais, culpabilizando-se a vítima e individualizando-se um problema de caráter coletivo e organizacional.

Nesse sentido, Souza (2013) apresenta e discute algumas importantes e recentes publicações a respeito da problemática relacionada aos chamados transtornos mentais $\mathrm{e}$ comportamentais relacionados ao trabalho e verifica que a Psicologia, como ciência e profissão, se encontra diante de inúmeros desafios concernentes tanto à formação dos profissionais, quanto a atuação deles nos mais diversos campos.

O autor destaca que o psicólogo, independente do lugar em que esteja atuando, poderá deparar-se com questões do processo saúde-doença em sua relação com o trabalho e que, independentemente do seu espaço de atuação e de quem o contrata, deve sempre colaborar preferencialmente de forma interdisciplinar e em equipes multiprofissionais - com a luta pela afirmação da vida e da saúde dos humanos, inclusive em seu trabalhar. Trata-se, aqui, de desafios para a Psicologia, que precisa instrumentalizar os psicólogos, desde a formação, para compreenderem as especificidades da relação trabalho e saúde-adoecimento, o que requer o desenvolvimento de um pensar-agir diferenciado, que remeta à consideração dos aspectos da organização, do processo e das condições de trabalho, da vivência subjetiva no trabalho e das repercussões para a saúde mental dos trabalhadores. Assim, é preciso que os psicólogos possam reconhecer

a subjetividade no trabalho, o significado que as pessoas atribuem a determinadas situações, o modo como cada um reage a partir de sua história de vida, de seus valores, das suas crenças, das suas experiências e das suas representações sobre a atividade desenvolvida. (SOUZA, 2013, p. 102).

Diante dos inúmeros desafios e da complexidade da questão da saúde mental relacionada ao trabalho, diversas pesquisas e publicações vêm sendo desenvolvidas no Brasil. Souza (2013) destaca algumas das mais recentes e importantes, como o dossiê específico sobre o tema, publicado em dois volumes pela Revista Brasileira de Saúde Ocupacional (RBSO, 2010; 2011) e intitulado "O mundo contemporâneo do trabalho e a saúde mental do trabalhador". 
O autor enfatiza que, nessa e em outras publicações científicas por ele citadas, há uma diversidade de temáticas abordadas, englobando estudos e pesquisas empíricas acerca de estresse, burnout, assédio moral, relação desemprego prolongado e saúde/adoecimento psicossocial, reabilitação profissional, relações de gênero, epidemiologia em saúde mental e trabalho, sentido do trabalho, discurso e prática corporativa etc. Os grupos pesquisados também variam, bem como "teorias, métodos e técnicas empregados para abarcar as suas singularidades e dos diferentes mundos do trabalho existentes" (SOUZA, 2013, p. 103).

Considerando o que foi exposto, justifica-se a relevância do projeto de extensão desenvolvido. Cabe ainda acrescentar que, tradicionalmente, a formação dos psicólogos não contempla, de modo suficiente, a relação entre trabalho, gestão e saúde mental, e muitos profissionais acabam por negligenciá-la em suas práticas.

Conforme o CFP/CREPOP (2008), a relação trabalho/subjetividade ainda não foi devidamente reconhecida nesse contexto, o que representa uma séria barreira para a atuação do psicólogo em todas as áreas, sobretudo na área de saúde do trabalhador. Daí infere-se que é importante que os estudantes de graduação em Psicologia tenham uma formação que contemple as relações entre a saúde e o adoecimento/sofrimento psíquico (ou os chamados transtornos mentais e comportamentais relacionados ao trabalho, conforme a linguagem dos órgãos governamentais).

Para uma atuação mais eficaz nesse campo, requer-se o aprimoramento dos conhecimentos, desde a formação, a fim de que os psicólogos possam utilizá-los nos diversos campos de atuação da Psicologia, cobrindo, ainda que parcialmente, uma enorme lacuna existente. A ampliação do escopo de compreensão e de atuação possibilita ao psicólogo a interação com outros profissionais da mesma área e também com os de outras especialidades afins, podendo, assim, trabalhar na promoção e na proteção da saúde mental dos trabalhadores, bem como no tratamento e na reabilitação dos casos de transtornos mentais relacionados ao trabalho.

No campo social mais amplo, verifica-se a necessidade de explanação dessa temática aos mais diversos atores sociais, tendo em vista a minimização (e até mesmo a negligência) em torno de sua importância e magnitude, conforme já apontado anteriormente.

Dados oficiais corroboram a importância dessa problemática na sociedade brasileira. Conforme o Ministério da Saúde (BRASIL, 2001), uma estimativa da Organização Mundial da Saúde (OMS) apontou que os transtornos mentais menores acometiam cerca de $30 \%$ dos trabalhadores ocupados, e os transtornos mentais graves, cerca de 5\% a 10\%. Dados mais recentes do Ministério da Previdência Social (BRASIL, 2012a) dão conta de que os transtornos mentais e comportamentais relacionados ao trabalho representam a terceira causa de afastamentos, ocupam terceiro lugar em quantidade de concessões de auxíliodoença acidentários e encontram-se na lista de acidentes de trabalho com códigos CID-10 mais incidentes, de acordo com a Classificação Internacional de Doenças (CID)

Esses dados evidenciam uma condição de sofrimento psíquico relacionado ao trabalho, que se reveste de extrema importância e complexidade, colocando um desafio ao campo "psi", ao campo das ciências em geral e para toda a sociedade. Assim, consideramos que devemos abordar, no mínimo, alguns questionamentos: como cuidar do trabalho de forma que não seja fator de adoecimento, e, sim, de produtividade, criatividade, saúde física e mental? Que trabalho queremos e devemos ter em nossa sociedade? Como desafiar a lógica perversa que 
conduz o trabalho, uma experiência decisiva e fundamental para os humanos, a se tornar um fator desestruturante, adoecedor e não um "fator de equilíbrio" (DEJOURS et al., 1993)?

Busca-se, com o projeto promover a participação efetiva de diversos segmentos da sociedade no debate, aprimorar o conhecimento e discutir formas de intervenção no âmbito das problemáticas que se referem às relações entre o trabalho e a subjetividade e suas repercussões no quadro de saúde-adoecimento psíquico dos trabalhadores. Dessa forma, busca-se intensificar a potência de agir da coletividade na promoção da saúde mental no trabalho, na prevenção e no tratamento adequado dos chamados transtornos mentais e comportamentais a ele relacionados.

O objetivo geral desdobra-se nos seguintes objetivos específicos: a) possibilitar o reconhecimento do vínculo entre os processos psicossociais e a saúde mental no trabalho; b) disseminar o conhecimento de abordagens teóricas e metodológicas que fundamentam a compreensão e as intervenções no campo da SMRT; c) realizar atividades que possibilitem o encontro entre os diferentes segmentos (profissionais, "gestores" e usuários) e o debate sinérgico entre eles acerca da temática SMRT; d) contribuir para o desenvolvimento de estratégias locais para a prevenção dos transtornos mentais e comportamentais relacionados ao trabalho.

\section{RESULTADOS}

A parceria firmada entre a universidade, o CEREST e o engajamento de professores, alunos, profissionais de saúde e educação e usuários do SUS no projeto de extensão universitária possibilitou a realização (até julho de 2014) das seguintes atividades:

1. Reuniões semanais da equipe para discussão de temas e textos referentes ao campo da SMRT, possibilitando aos alunos um aprimoramento do conhecimento acerca dessa temática, com repercussões positivas na qualidade de sua formação;

2. 11 de abril de 2014: participação da equipe no Encontro Regional de Saúde do Trabalhador: a) exposição e apresentação do pôster do projeto pelas alunas que compõem a equipe; b) apresentação do projeto pelas alunas por meio de palestra e exposição de slides; c) palestra sobre "Desenvolvimento socioeconômico e seus reflexos na saúde (mental) do trabalhador e da trabalhadora", proferida pelo coordenador do projeto;

3. 22 de maio de 2014: exposição do pôster do projeto no Encontro Macrorregional de Saúde do Trabalhador;

4. 5 de junho de 2014: realização da oficina "Dialogando sobre as relações entre saúde mental e trabalho";

5. 31 de julho de 2014: apresentação do projeto e palestra sobre a saúde mental relacionada ao trabalho para alunos de curso técnico em enfermagem do trabalho.

Ao final da oficina, realizada em 5 de junho de 2014, foi distribuído aos participantes um formulário para avaliação (Figura 1). 
Figura 1 - Formulário de avaliação da oficina "Dialogando sobre as relações entre saúde mental e trabalho.

Pedimos a sua gentil colaboração na avaliação da Oficina "Dialogando sobre as relações entre saúde mental e trabalho", realizada hoje (5/06/2014), respondendo às questões abaixo:

1) Você considera que o tema abordado na Oficina foi:

( ) EXCELENTE; ( ) MUITO BOM; ( ) BOM; ( ) REGULAR;

( ) INSUFICIENTE

2) Quanto ao modo como o tema foi abordado pelos instrutores, você considera:

( ) EXCELENTE; ( ) MUITO BOM; ( ) BOM; ( ) REGULAR;

( ) INSUFICIENTE

3) Qual sua opinião sobre os materiais utilizados na Oficina?

( ) EXCELENTE; ( ) MUITO BOM; ( ) BOM; ( ) REGULAR;

( ) INSUFICIENTE

4) Quanto aos recursos didáticos e dinâmicas utilizados, você considera:

( ) EXCELENTE; ( ) MUITO BOM; ( ) BOM; ( ) REGULAR;

( ) INSUFICIENTE

5) Em relação ao cumprimento do objetivo da Oficina, que grau você atribui?

( ) EXCELENTE; ( ) MUITO BOM; ( ) BOM; ( ) REGULAR;

( ) INSUFICIENTE

Críticas e sugestões (opcional):

Seu nome (opcional):

Muito obrigado!

Fonte: Os autores (2014).

A análise das respostas possibilitou à equipe verificar que a atividade foi avaliada positivamente pelos participantes, pois a maioria das respostas aos cinco itens avaliados foi "excelente" ou "muito bom", graus máximos da avaliação, como verificado na Figura 1.

Além disso, no item "Críticas e sugestões" alguns participantes demandaram a realização de outras atividades e mais tempo de duração para cada uma, sinalizando que houve receptividade, interesse e disponibilidade para manutenção e aprimoramento do debate proposto.

No que concerne ao aproveitamento acadêmico dos alunos participantes do projeto, uma das alunas relatou que as experiências vivenciadas nos encontros, voltados para públicos diversos, proporcionaram uma visão crítica sobre como o campo da SMRT precisa lidar com o desconhecimento e/ou negligência das organizações e da sociedade acerca da importância do papel do próprio trabalhador como detentor de conhecimentos sobre o seu processo de trabalho e sobre as suas repercussões no processo de saúde-adoecimento. Além disso, ressaltou que os textos utilizados para estudos foram essenciais para o aprofundamento da compreensão da temática abordada, auxiliando, então, nas palestras, apresentações e demais atividades das quais participou. 
Outra aluna, por sua vez, relatou que o projeto tem contribuído para a sua formação, pois possibilita contato direto com um assunto de extrema importância - a saúde mental do trabalhador -, muito negligenciado pela sociedade. Afirma que, por mais que esse tema seja essencial para a formação do psicólogo, pouco se discute sobre ele nas salas de aula. Outro ponto benéfico apontado por ela é estar em parceria com profissionais do CEREST, pois isso permite ampliar seus conhecimentos diante das experiências, opiniões e rico conhecimento desses profissionais que trabalham diretamente com a saúde do trabalhador. Espera que o projeto ainda proporcione mais conhecimentos e "bagagem", para que possa atuar da melhor maneira como psicóloga, sempre levando em consideração aspectos do trabalho, em qualquer área que decida seguir.

\section{CONSIDERAÇÕES FINAIS}

Discutiu-se a importância das temáticas e das abordagens compreendidas no campo da SMRT, bem como a magnitude da problemática a ela relacionada e a relevância social da discussão, ampliando-a, cada vez mais, com a inclusão de diversos atores.

Identificou-se uma lacuna tanto no que concerne ao campo social mais amplo a respeito da insuficiência de conhecimentos e de estratégias eficazes de intervenção, assim como apontouse uma lacuna também existente na formação dos alunos de Psicologia. Diante dessa realidade, propôs-se o projeto de extensão universitária, que foi detalhadamente apresentado neste texto.

Tomando como parâmetros as avaliações dos participantes do projeto e dos participantes das atividades, colhidas em conversas, do formulário de avaliação e, também, contando com a avaliação da equipe após cada atividade realizada, pode-se inferir que o projeto vem sendo bem recebido pela comunidade a qual se destina e vem tendo repercussões positivas na formação dos alunos. Também as participações nos encontros permitiram a divulgação do projeto em eventos que têm repercussão social e científica.

Assim, com o desenvolvimento do projeto, tem-se verificado um incremento do interesse e do conhecimento sobre a temática por parte de todos os envolvidos, à medida que o debate se espraia e que informações são transmitidas sobre sua importância e sobre sua repercussão psicossocial.

\section{REFERÊNCIAS}

ATHAYDE, M. Saúde mental e trabalho: questões para discussão no campo da saúde do trabalhador. In: MINAYO-GOMEZ et al. Saúde do trabalhador na sociedade brasileira contemporânea. Rio de Janeiro: Fiocruz, 2011.

BRASIL. Ministério da Previdência Social. Anuário Estatístico da Previdência Social, 2012. Brasília, DF: MPS/DATAPREV, 2012a.

Portaria $\mathrm{n}^{\mathrm{o}} 2.728$, de 11 de novembro de 2009. Dispõe sobre a Rede Nacional de Atenção Integral à Saúde do Trabalhador (RENAST) e dá outras providências. Brasília, DF: Ministério da Saúde do Brasil, 2009. 
Portaria $\mathrm{n}^{0}$ 1.823, de 23 de agosto de 2012. Institui a Política Nacional da Saúde do Trabalhador e da Trabalhadora. Brasília, DF: Ministério da Saúde do Brasil, 2012b.

CFP - Conselho Federal de Psicologia. Psicologia crítica do trabalho na sociedade contemporânea. Brasília: Conselho Federal de Psicologia, 2010.

CREPOP - Centro de Referência Técnica em Psicologia e Políticas Públicas. Saúde do trabalhador no âmbito da saúde pública: referências para a atuação do psicólogo. Brasília, DF: Conselho Federal de Psicologia, 2008.

DEJOURS, C. Addendum: da psicopatologia à psicodinâmica do trabalho. In: LANCMAN, S.; SZNELWAR, L. (Org.). Christophe Dejours: da psicopatologia à psicodinâmica do trabalho. Rio de Janeiro: Fiocruz; Brasília: Paralelo 15, p. 47-104, 2004.

Trabalho vivo: Tomo I, sexualidade e trabalho. Brasília: Paralelo 15, 2012.

DEJOURS, C.; DESSORS, D.; DESRIAUX, F. Por um trabalho fator de equilíbrio. Revista de Administração de Empresas, São Paulo, v. 33, n. 3, p. 98-104, maio/jun. 1993.

MENDES, R.; DIAS, E. C. Da medicina do trabalho à saúde do trabalhador. Revista de Saúde Pública, São Paulo, v. 25, n. 5, p. 341-349, 1991.

PAPARELLI, R.; SATO, L.; OLIVEIRA, F.. A saúde mental relacionada ao trabalho e os desafios aos profissionais de saúde. Revista Brasileira de Saúde Ocupacional, São Paulo, v. 36, n. 123, p. 118-127, 2011.

RBSO - Revista Brasileira de Saúde Ocupacional. Dossiê: O mundo contemporâneo do trabalho e a saúde mental do trabalhador - I. 2010. Disponível em: $<$ http://www.fundacentro.gov.br/arquivos/ rbso/RBSO-122-vol-35-completo.pdf $>$. Acesso em: 20 jun. 2014.

O mundo contemporâneo do trabalho e a saúde mental do trabalhador - II. 2011; Acesso em: <http://www.fundacentro.gov.br/rbso/dossie-tematico-saude-mental-do-trabalha $>$. Acesso em: 20 jun. 2014.

SELIGMANN-SILVA, E. Trabalho e desgaste mental: o direito de ser dono de si mesmo. São Paulo: Cortez, 2011.

SELIGMANN-SILVA, E. et al. Editorial. Revista Brasileira de Saúde Ocupacional, São Paulo, v. 35 n. 122, p. 185-186, 2010a.

SELIGMANN-SILVA, E. et al. Apresentação. Revista Brasileira de Saúde Ocupacional, São Paulo, v. 35 n. 122, p. 187-191, 2010 b.

SOUZA, W. F. Transtornos mentais e comportamentais relacionados ao trabalho: o que a Psicologia tem a dizer e a contribuir para a saúde de quem trabalha? Fractal Revista de Psicologia, Niterói, v. 25, n. 1, p. 99-108, jan.-abr. 2013.

Submetido em 25 de agosto de 2014.

Aprovado em 15 de setembro de 2014. 